\title{
Immunofluorescent localization of Tamm-Horsfall mucoprotein in human kidney
}

\author{
J. K. McKENZIE ${ }^{1}$ AND E. G. McQUEEN \\ From the Wellcome Medical Research Institute, Department of Medicine, and the Department of Pharmacology \\ University of Otago Medical School, Dunedin, New Zealand
}

SYNOPSIS A fluorescein-labelled antiserum to human Tamm-Horsfall mucoprotein applied to frozen human kidney sections gave strong specific labelling, mainly of cells of tubules in the outer $\underset{N}{N}$ medulla. By comparison with adjacent serial sections stained for alkaline phosphatase and succinic $\omega$ dehydrogenase, it is suggested that material reacting immunologically as Tamm-Horsfall muco- $\omega_{ \pm}$ protein is found particularly in the cells of the ascending limb of the loop of Henle and the macula densa segment of the distal tubule.

The urinary mucoprotein first described by Tamm and Horsfall in 1950 appears to originate in the kidney. It has been positively identified only in kidney cortex and medulla (Keutel, King, and Boyce, 1964) although reactions which may be non-specific have been described in tissues such as lung, colon, parotid gland, skin, fat, and striated muscle (Boyce, King, and Fielden, 1961 ; Cornelius, Mia, and Rosenfeld, 1965). McQueen $(1962,1966)$ has shown that hyaline urinary casts which arise within the kidney are almost entirely composed of Tamm-Horsfall mucoprotein. Radioactive tracer work shows that this mucoprotein is not formed from plasma proteins but appears to be synthesized in the kidney (Cornelius et al, 1965).

The site of origin in the kidney is presently in dispute. Keutel (1965), using an immunofluorescent technique, claims that the proximal tubule is the only region where 'uromucoid' is demonstrably present. (Uromucoid is a urinary material isolated in a slightly different way from the classical salt precipitation technique of Tamm and Horsfall, but which is very similar to, and when purified, is probably identical with, Tamm-Horsfall mucoprotein.) Friedmann (1966), however, reports greatest fluorescence with a Tamm-Horsfall antiserum in distal and collecting tubules and perhaps the loop of Henle but none in the proximal tubule.

The present experiments also use an immunofluorescent technique and demonstrate greatest fluorescence in the cells of the ascending limb of the loop of Henle and the distal tubule as far as the macula densa region.

'Present Address: Department of Medicine, Medical College, Winnipeg, Manitoba.

Received for publication 29 August 1968.
MATERIALS AND METHODS

Human Tamm-Horsfall mucoprotein was prepared by precipitation from fresh urine with $0.58 \mathrm{M}$ sodium ${ }^{\circ}$ chloride and purified by twice redissolving in distilled water and reprecipitating with $0.58 \mathrm{M}$ salt. A solution containing $10 \mathrm{mg} / \mathrm{ml}$ was mixed with an equal volume of Freund's complete adjuvant and $4 \mathrm{ml}$ of the mixture injected intramuscularly into a sheep twice at fortnightly $\frac{\mathrm{D}}{\mathbb{2}}$ intervals. Further injections of $2 \mathrm{ml}$ of the Tamm- $\cong$ Horsfall mucoprotein alone were given intraperitoneally $\overrightarrow{\overrightarrow{0}}$ and finally intravenously at two-day intervals for a total 3 of seven injections. After two years two further intravenous injections of $10 \mathrm{ml}$ of antigen were given at: weekly intervals and the animal was bled a week later.

The serum proteins then contained a large proportion 0 $(43 \%)$ of gamma globulin. This was precipitated and washed twice with $40 \%$ saturated ammonium sulphate 3 at $4^{\circ} \mathrm{C}$, taken up in distilled water and dialysed until free ${ }_{0}$ of sulphate. Polyacrylamide gel electrophoresis at this stage showed only a gamma globulin band. The gamma globulin was conjugated with fluorescein isothiocyanate $\supset$ $(0.05 \mathrm{mg} / \mathrm{mg}$ protein) according to the method of Nairn (1962). Unconjugated fluorescein was removed by repeated dialysis against $0.06 \mathrm{M}$ phosphate buffer $p \mathrm{H} \mathrm{7.4N}$ and gel filtration using Sephadex G100.

Both the conjugated and the unconjugated gamma $N$ globulin preparations gave single precipitin lines against Tamm-Horsfall mucoprotein using Ouchterlony $\omega$ gel diffusion but the conjugated gamma globulin appeared to diffuse more easily than the unconjugated. The con- - ? jugated gamma globulin was adsorbed initially with $\Phi_{\overparen{D}}$ mouse liver powder and, just before use, with rat kidney? homogenate. The adsorbed antiserum gave strong 0 fluorescence with human Tamm-Horsfall mucoprotein and human hyaline casts but almost no staining of rat kidney, suggesting that the Tamm-Horsfall antibody is specific for human Tamm-Horsfall mucoprotein.

Fresh human kidney was obtained either by percu +2 taneous biopsy in cases where there proved to be only? 334 
minor glomerular disturbances, or at nephrectomy for renal carcinoma or renal trauma. Small pieces were quenched directly in liquid nitrogen. Cryostat sections 7 to $11 \mu$ thick were cut serially and placed on dry slides or cover slips at room temperature. One section in three was flooded with the fluorescein-conjugated antiserum and allowed to remain in contact with it for 40 minutes at room temperature in humid conditions. After this the antiserum was gently removed with successive washes of $0.1 \mathrm{M}$ phosphate buffer $p \mathrm{H} 7.4$ containing $0.5 \mathrm{M}$ sodium chloride to prevent solution of Tamm-Horsfall mucoprotein in the sections, which were then mounted under sealed coverslips in the same buffer. Some sections were first treated with unlabelled antiserum for 40 minutes in the same conditions, washed with phosphate saline, and treated as above with labelled antiserum.

Intervening sections were alternately stained for alkaline phosphatase and succinic dehydrogenase or, on occasion, with haemotoxylin and eosin.

The method of Gomori (Pearse, 1960) was used to stain for alkaline phosphatase except that the sections were first fixed in ethanol for 10 minutes and the final stain used was $1 \%$ safranin. For succinic dehydrogenase the method according to McManus and Mowry (1960) was used but incorporating nitro-blue tetrazolium
$(1 \mathrm{mg} / \mathrm{ml})$ instead of tetrazolium blue. The sections were examined in a Reichert Zetopan microscope using dark ground ultraviolet illumination $\left(E_{2}\right.$ exciter and $\mathrm{SP}_{2}$ barrier filters) for the fluorescein-treated sections.

\section{RESULTS}

'Blocking' of the fluorescent antibody stain by prior application of unconjugated antibody is demonstrated in Figs. 1 and 2 (not serial sections). The sections show medullary ray tissue taken from the same biopsy specimen treated simultaneously as described with the same camera exposures and, as far as possible, similar illumination. The 'unblocked' section shows uniformly fluorescent tubules with even cytoplasmic staining and unstained nuclei contrasting strongly with the surrounding nonfluorescent tissue. The 'blocked' specimen shows almost no fluorescence, the small degree present being spread over the whole field and presumably due to non-specific staining or autofluorescence.

In human kidney sections from operation specimens bright fluorescence was present intracellularly,

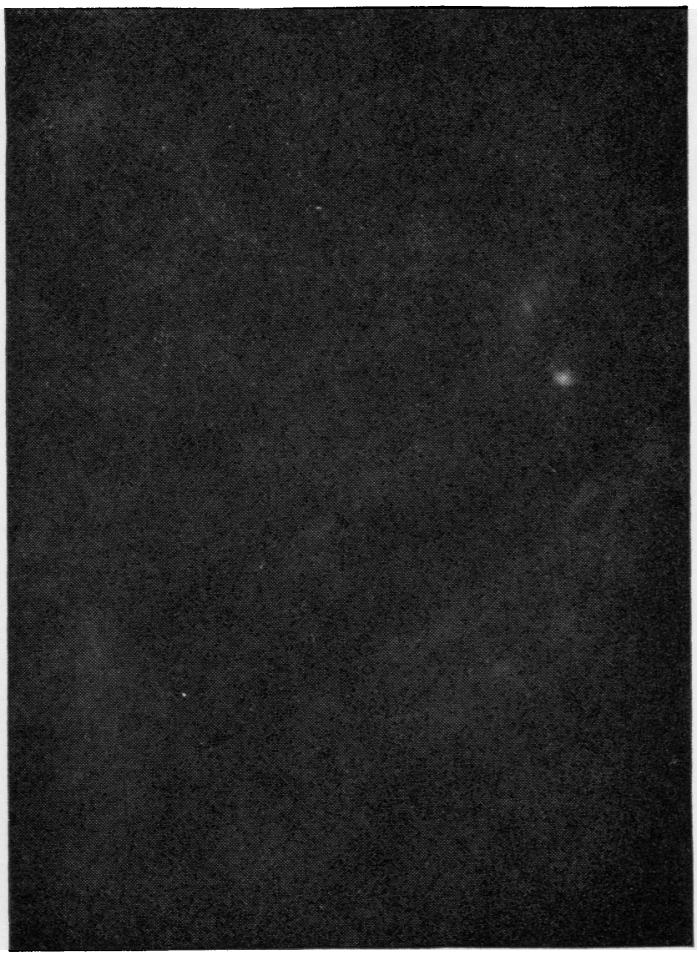

FIG. 2.
FIG. 1.

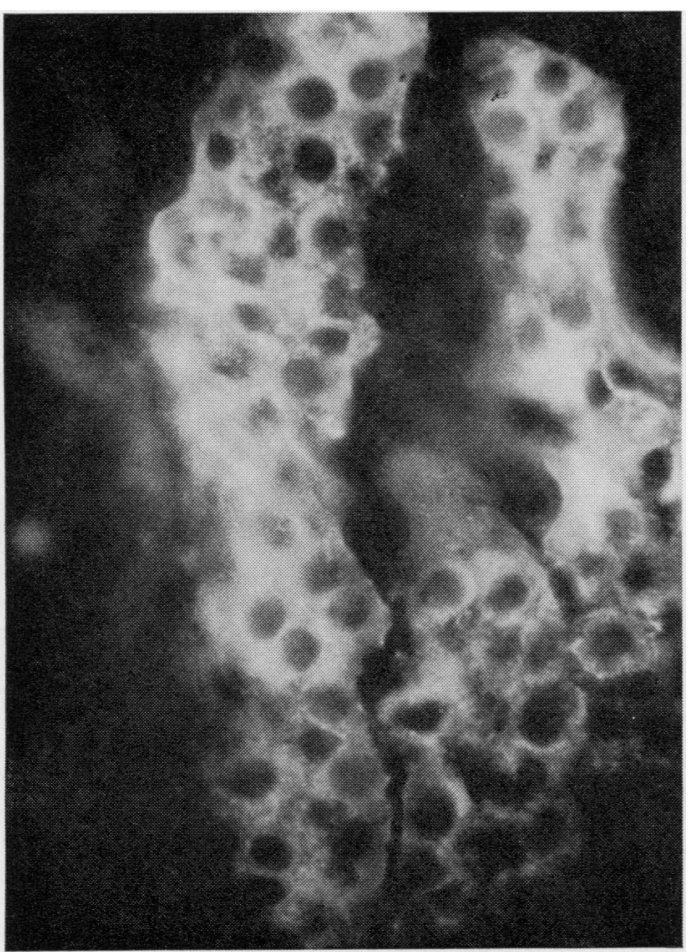

FIG. 1. Section of medullary ray treated with fluorescent antiserum. $\times 670$.

FIG. 2. Similar (not serial) section of medullary ray pretreated with unconjugated antiserum before treatment with fluorescent antiserum. $\times 670$. 


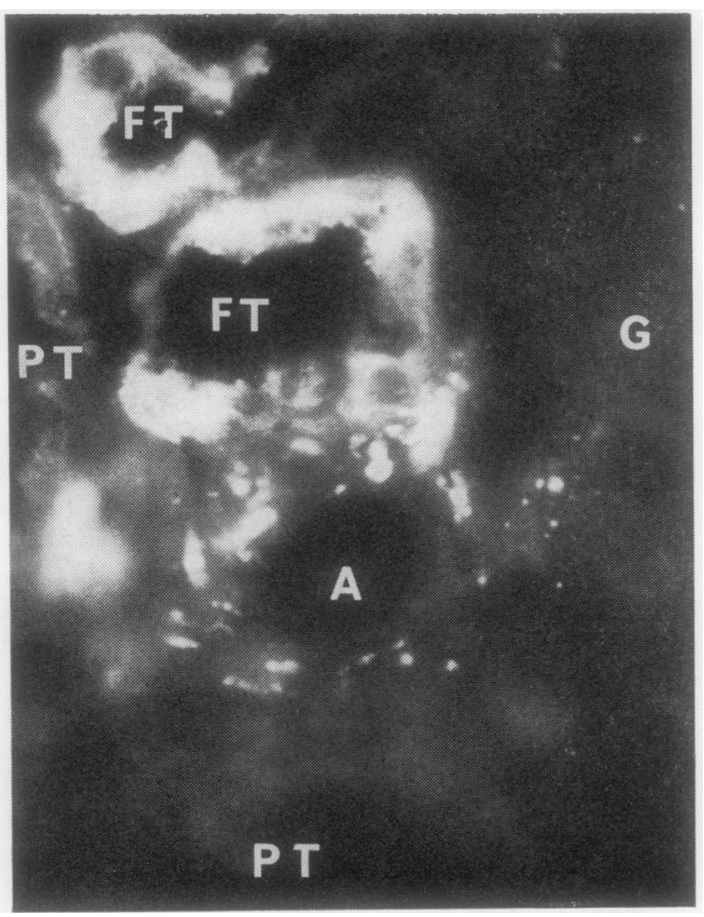

FIG. 3.

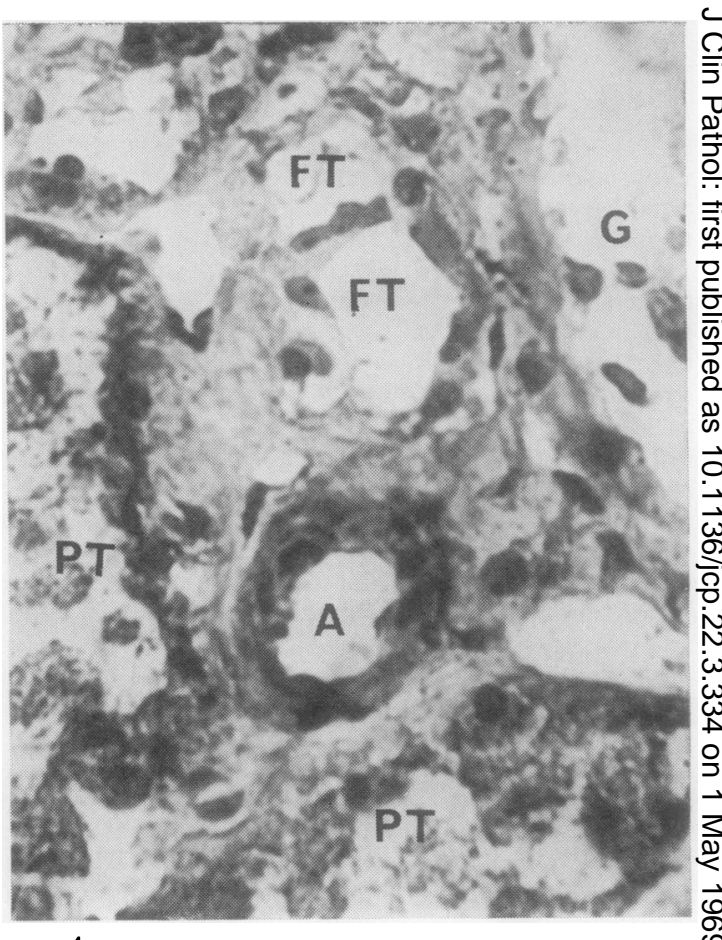

FIG. 4.

FIG. 3. Fluorescent tubules $(F T)$ beside arteriole $(A)$ and glomerulus $(G)$ contrasting with non-fluorescent proximal tubules $(P T) . \times 670$

FIG. 4. Cells of proximal tubules (PT) show apical staining for alkaline phosphatase. Fluorescent tubules (FT) do not show such staining. $\times 670$. (Serial section to Figure 3 .)

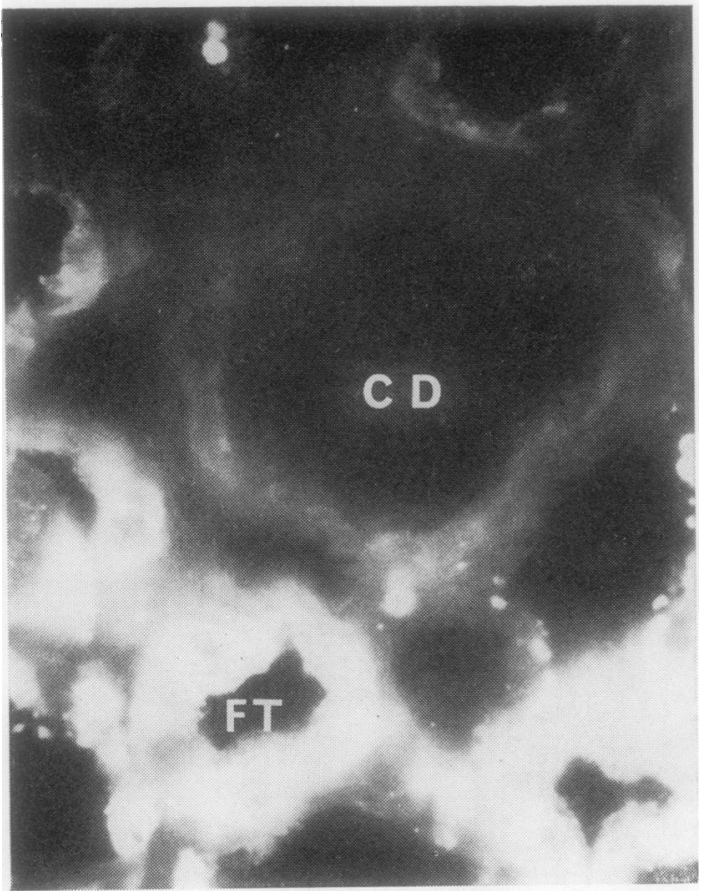

FIG. 5.

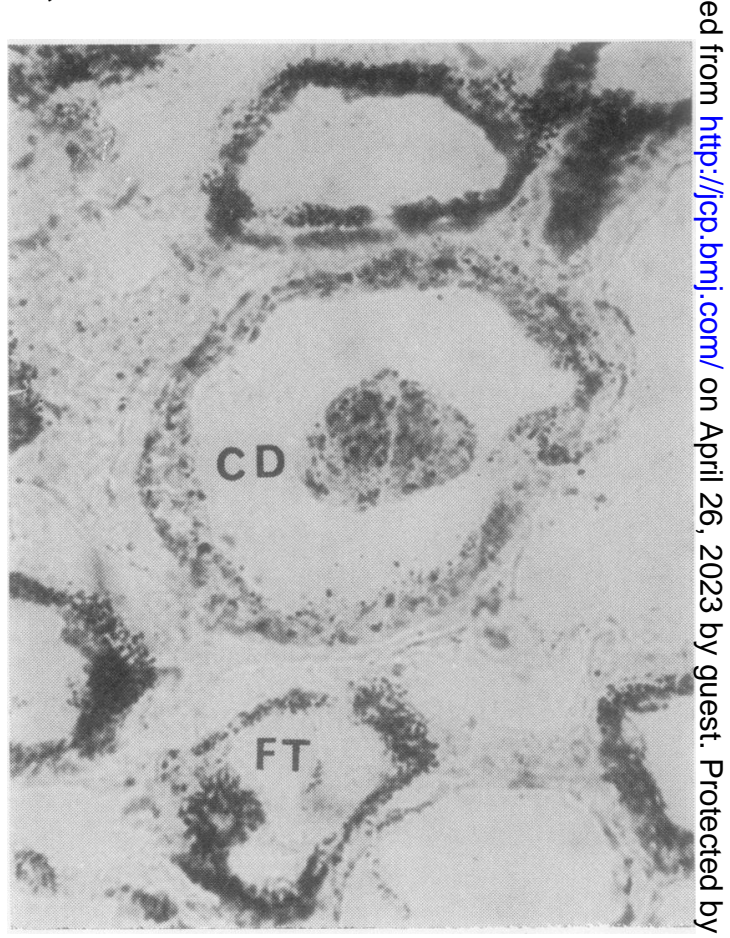

FIG. 6

FIG. 5. Medullary tissue shows central non-fluorescent tubule, probably collecting duct (CD), contrasting wit strongly fluorescent tubules (illumination is uneven). $\times 670$.

FIG. 6. Serial section stained for succinic dehydrogenase. Fluorescent tubules (FT), presumed to be ascending limbs of loops of Henle stain heavily for succinic dehydrogenase. Unstained thin tubules surrounding collecting duct, either descending limbs or vasa rectae, are not fluorescent in Figure 5. 

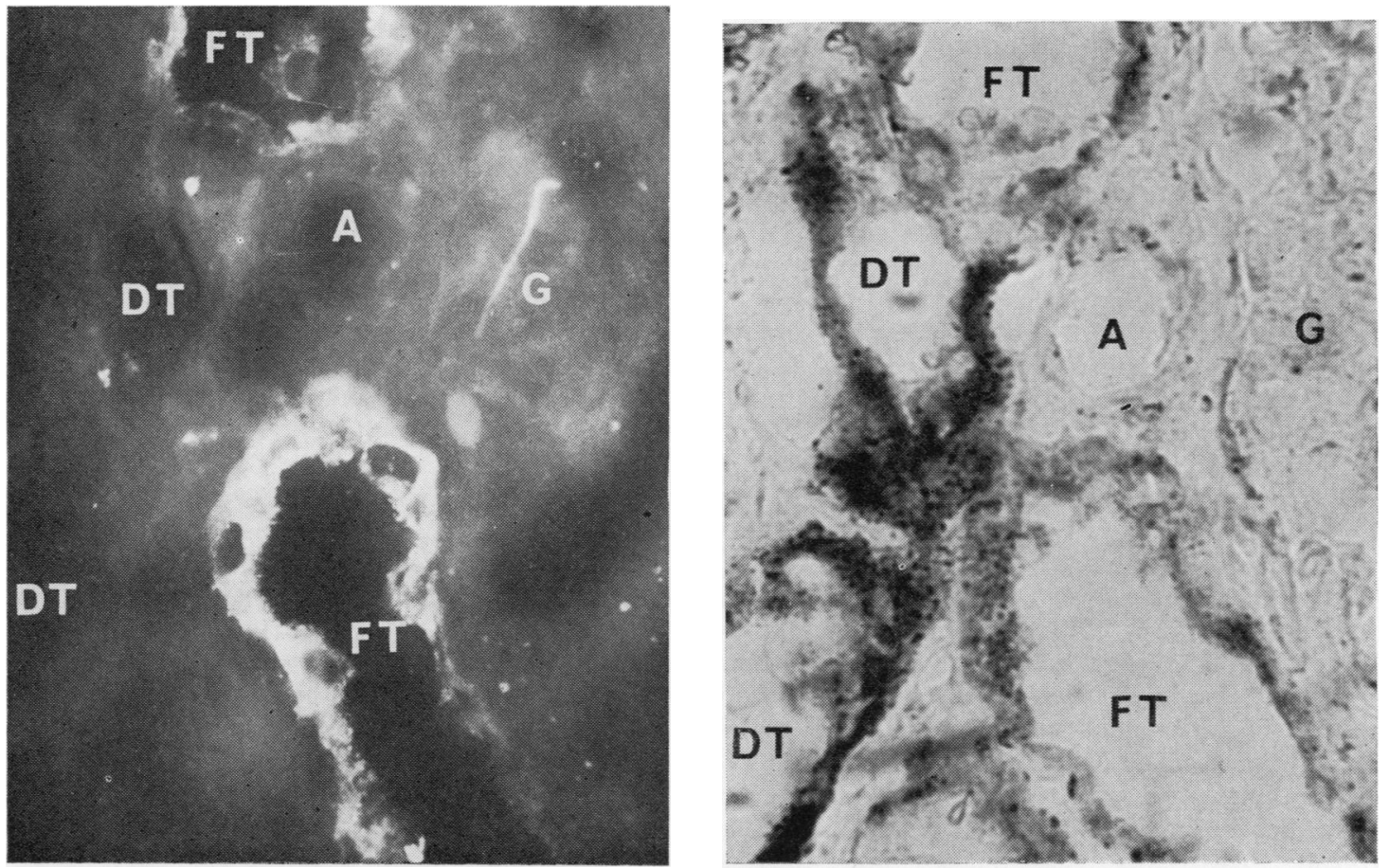

FIG. 7.

FIG. 8.

FIG. 7. Fluorescent tubules $(F T)$ surround arteriole $(A)$ beside glomerulus $(G) . \times 670$.

FIG. 8. Distal tubules (DT) staining heavily for succinic dehydrogenase are not fluorescent in Figure $7 . \times 670$. (Serial section to Figure 8.)

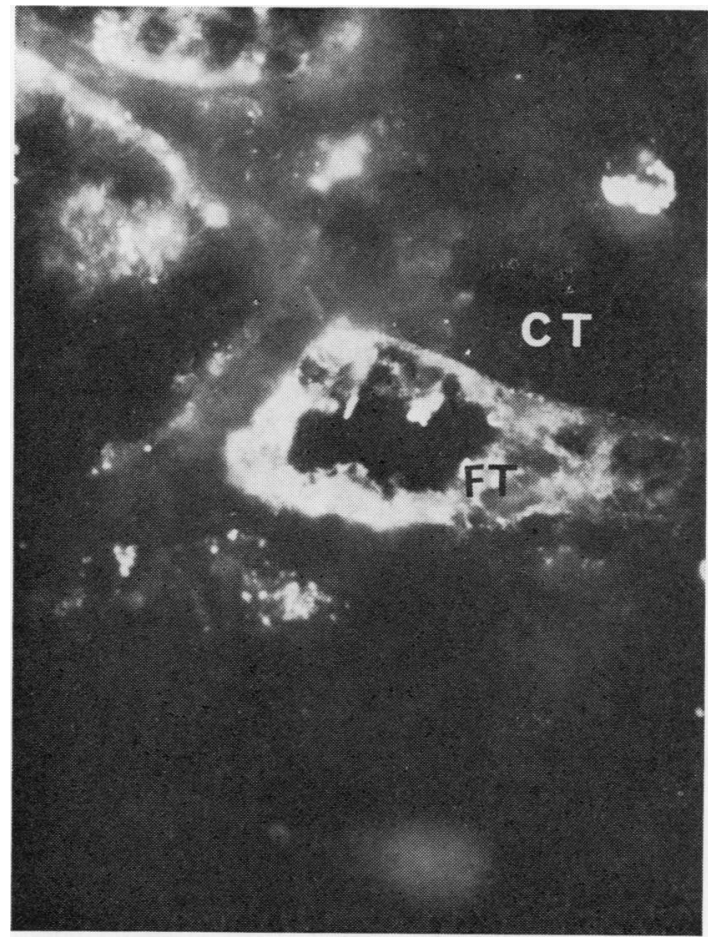

FIG. 9.

FIG. 9. Fluorescent tubule in outer medulla $(F T) . \times 670$.

FIG. 10. Collecting tubule (CT) in contiguous serial section stained with haematoxylin and eosin is not fluorescent in Figure 9. $\times 670$. 
particularly in certain tubules in the outer medulla and in medullary rays. In the cortex there were occasional fluorescent tubules usually closely related to glomeruli (Figs. 3 and 7). The glomeruli themselves were not fluorescent.

In comparison with adjacent serial sections stained for alkaline phosphatase, which is concentrated in the brush borders of proximal tubule cells, there appeared to be little or no fluorescence in proximal tubules (Figs. 3 and 4). Succinic dehydrogenase, according to Wachstein (1955), is located particularly in cells of the ascending limbs of the loops of Henle and in distal tubules, to a lesser extent in proximal tubules, to a minor degree only in collecting tubules, and not at all in the descending limbs of the loops of Henle. By comparing serial sections it could be seen that fluorescing tubules in the medulla and medullary rays stained heavily for succinic dehydrogenase (Figs. 5 and 6). In the cortex, however, convoluted tubules which were heavily stained for succinic dehydrogenase (presumably distal tubules) did not fluoresce (Figs. 7 and 8). Cortical fluorescent tubules were often seen to be in close association with arterioles near glomeruli (Figs. 3 and 7). In an occasional section, closely packed, regularly arranged nuclei could be seen in the side of the fluorescing tubule related to the arteriole suggesting that these represented macula densa segments.

Collecting tubules were relatively easily identified by the simple cuboidal appearance of their cells which have particularly obvious cell boundaries. Figures 9 and 10 show a haematoxylin-and-eosinstained section of medullary ray and the contiguous fluorescein-treated section. The brightly fluorescent tubule may be recognized by its shape in the corresponding haematoxylin-and-eosin-stained section. It can be seen that the adjacent tubule above it is a collecting tubule which does not fluoresce. No examples of small collecting tubules or of larger ducts showing intracellular fluorescence have been found.

\section{DISCUSSION}

Evidence for the specificity of the reaction with the labelled antiserum was provided by the ability to 'block' specific staining by prior treatment with unconjugated antiserum. Adsorption with rat kidney homogenate appeared to reduce non-specific fluorescence very markedly, producing high contrast between specifically stained areas and the rest of the kidney. We were fortunate in that there does not appear to be marked cross-specificity between rat and human Tamm-Horsfall mucoprotein. We are thus confident that the conjugated anti-Tamm-
Horsfall antiserum is specifically staining materiat which reads immunologically as Tamm-Horsfa类 mucoprotein.

The failure to obtain significant fluorescence i⿱艹 tubules staining for alkaline phosphates is stron: evidence against the existence of a high concentro tion of Tamm-Horsfall mucoprotein in proxima tubule cells. These results conflict with those of Keutel (1965) but the methods of preparing sections and of labelling the antiserum differ. In particular the antiserum of Keutel does not appear to have been adsorbed with either a tissue powder or a homogen: ate, although it is claimed that adjustment of $p+4$ in his method greatly reduces non-specific protein binding. We have noted some tendency of proximab tubules to fluoresce when the antiserum has nof been freshly treated with homogenized rat kidnef. As this is a variable phenomenon we have regarded it as being due to non-specific staining. Friedman $\vec{n}$ (1966), using a similar antiserum preparation to ours, has also found no specific proximal tubular fluorescence. He states that cells of the distal and collecting tubules (and perhaps the loops of Henles showed specific fluorescence. In the present experiment it was apparent that tubules in the corte staining heavily for succinic dehydrogenase did not fluoresce with the specific antiserum. In medullary rays, however, tubules staining heavily for succing dehydrogenase did fluoresce. This suggests that is the cells of the ascending limb of the loop of Henle rather than the cortical distal tubule which contain Tamm-Horsfall mucoprotein. Further, the frequent association of fluorescing tubules with: afferent arterioles and the nuclear arrangemen sometimes seen makes it likely that the cells of the macula densa segment contain Tamm-Horsfä. mucoprotein. Unlike Friedmann, we have nevê found intracellular fluorescence in collecting tubule

The significance of the localization of Tamn? Horsfall mucoprotein in the ascending limb of the loop of Henle and macula densa region is obscure but it is interesting that the outer medullary portion of the ascending limb is the section of the tubuR associated with sodium reabsorption unaccompanied by water.

The presence of high concentrations of Tammo Horsfall mucoprotein in the ascending limb cells suggests that it may be secreted into the tubulas lumen in this region. Aggregation into hyalirfe casts could then occur with increasing solute and hydrogen ion concentration in the distal convoli $\vec{\Phi}$ tions and particularly in the collecting tubules.

An additional point of practical histologica interest is that the antiserum appears to provide $\frac{O}{2}$ method of identification of the macula densa portion of the tubule in cortical sections and of the 
ascending limb of the loop of Henle in the outer medulla.

We wish to thank Miss J. Ledingham and $\mathrm{Mr}$ M. Birtles for expert technical assistance, and Professors W. E. Adams and A. Wynn Williams for use of their cryostat equipment. We are indebted to the Medical Research Council of New Zealand and the Golden Kiwi Medical Research Distribution Committee for financial support.

\section{REFERENCES}

Boyce, W. H., King, J. S., Jr, and Fielden, M. L.(1961). J. clin. Invest., 40, 1453.
Cornelius, C. E., Mia, A. S., and Rosenfeld, S. (1965). Invest. Urol., 2, 453.

Friedmann, T. (1966). Experientia (Basel), 22, 624.

Keutel, H. J. (1965). J. Histochem. Cytochem., 13, 155.

, King, J. S., Jr, and Boyce, W. H. (1964). Urol. int. (Basel), 17, 324.

McManus, J. F. A., and Mowry, R. W. (1960). Staining Methods: Histologic and Histochemical 1 st ed., p. 188. Hoeber, New York. McQueen, E. G. (1962). J. clin. Path., 15, 367.

, (1966). Lancet, 1, 397.

Nairn, R. C. (1962). Fluorescent Protein Tracing, p. 21. Livingstone, Edinburgh.

Pearse, A. G. E. (1960). Histochemistry Theoretical and Applied 2nd ed., p. 868. Churchill, London.

Wachstein, M. (1955). J. Histochem. Cytochem., 3, 246. 